\title{
SOME OBSERVATIONS AND SPECULATIONS ON PARTITIONS INTO $d$-TH POWERS
}

\author{
MACIEJ ULAS (1) \\ (Received 14 December 2020; accepted 20 December 2020; first published online 28 January 2021)
}

\begin{abstract}
The aim of this article is to provoke discussion concerning arithmetic properties of the function $p_{d}(n)$ counting partitions of a positive integer $n$ into $d$ th powers, where $d \geq 2$. Apart from results concerning the asymptotic behaviour of $p_{d}(n)$, little is known. In the first part of the paper, we prove certain congruences involving functions counting various types of partitions into $d$ th powers. The second part of the paper is experimental and contains questions and conjectures concerning the arithmetic behaviour of the sequence $\left(p_{d}(n)\right)_{n \in \mathbb{N}}$, based on computations of $p_{d}(n)$ for $n \leq 10^{5}$ for $d=2$ and $n \leq 10^{6}$ for $d=3,4,5$.
\end{abstract}

2020 Mathematics subject classification: primary 11P83; secondary 05A17.

Keywords and phrases: identities, numerical computations, partitions into powers.

\section{Introduction}

Given $A \subset \mathbb{N}_{+}$and $n \in \mathbb{N}$, a partition of a nonnegative integer $n$ with parts in $A$ is any representation of $n$ in the form

$$
n=a_{1}+\cdots+a_{k},
$$

where $a_{i} \in A$. Two partitions that differ only in the order of their summands are considered to be the same and we can assume that $a_{1} \geq a_{2} \cdots \geq a_{k}$. In particular, if $A=\mathbb{N}_{+}$, then the number of partitions with parts in $\mathbb{N}_{+}$is denoted by $p(n)$, the famous partition function introduced by Euler and extensively studied by Ramanujan.

The literature on arithmetic properties of functions counting various types of partitions is enormous. However, the theory concentrates mainly on the case when the set $A$ is a sum of disjoint arithmetic progressions. In this case, the theory is especially rich because of the connections with modular forms and the general theory of $q$ series (see, for example, [2]). In this case, the counting function $A(x)=\#\{a \in A: a \leq x\}$ is linear, that is, $A(x)=O(x)$. There is also a nice theory connected with the set of powers

The research of the author is supported by the grant of the National Science Centre (NCN), Poland, No. UMO-2019/34/E/ST1/00094.

(c) Australian Mathematical Publishing Association Inc. 2021. This is an Open Access article, distributed under the terms of the Creative Commons Attribution licence (http://creativecommons.org/ licenses/by/4.0/), which permits unrestricted re-use, distribution, and reproduction in any medium, provided the original work is properly cited. 
of a fixed integer $m$ (so-called $m$-partitions); in this case, $A(x)$ has logarithmic growth, that is, $A(x)=O(\log x)$.

On the other hand, very little is known about the arithmetic behaviour of partition functions counting partitions into $d$ th powers, where $d \in \mathbb{N}_{\geq 2}$ is fixed. In this case, $A=\left\{k^{d}: k \in \mathbb{N}\right\}$ and the growth of $A(x)$ is $O\left(x^{1 / d}\right)$, which is between the two cases mentioned earlier. Let $p_{d}(n)$ denote the number of partitions of $n$ into $d$ th powers. From general principles, the ordinary generating function of the sequence $\left(p_{d}(n)\right)_{n \in \mathbb{N}}$ has the form

$$
P_{d}(q)=\sum_{n=0}^{\infty} p_{d}(n) q^{n}=\prod_{n=1}^{\infty} \frac{1}{1-q^{n^{d}}} .
$$

Up to now, the main line of research has been to investigate the asymptotic behaviour of $p_{d}(n)$. Hardy and Ramanujan claimed in [6], and Wright proved in [10], that

$$
\log p_{d}(n) \sim(d+1)\left(\frac{1}{d} \Gamma\left(1+\frac{1}{d}\right) \zeta\left(1+\frac{1}{d}\right)\right)^{d /(d+1)} n^{1 / d} .
$$

Wright's very complicated proof was simplified by Vaughan in the case $d=2$ (see [9]) and in the general case by Gafni (see [5]). The proofs of Vaughan and Gafni are based on the circle method. A new proof, using only the saddle point method, was presented by Tenenbaum et al. (see [8]). To the best of our knowledge, apart from identities between partitions into $d$ th powers of various types, which can be deduced from simple manipulations of infinite products, and a recent result of Ciolan (see [3]), who proved that the number of partitions into squares with an even number of parts is asymptotically equal to that of partitions into squares with an odd number of parts, there are no theoretical or experimental results. The absence of such results was the main motivation for our research.

In Section 2, we prove some congruences for functions counting various types of partitions into $d$ th powers, where $d \in \mathbb{N}_{\geq 2}$. In particular, if $A_{2, p_{2}}(n)$ denotes the number of partitions into $d$ th powers of integers not divisible by $2^{d}$ or $p_{2}^{d}$ and $B_{2, p_{2}}(n)$ denotes the number of partitions of $n$ into distinct $d$ th powers not divisible by $p_{2}^{d}$, where each part has one among $2^{d}-1$ colours, then $A_{2, p_{2}}(n) \equiv B_{2, p_{2}}(n)(\bmod 2)$.

In Section 3, we present many computational observations based on our computer experiments. In particular, we state several questions and conjectures concerning the arithmetic behaviour of the sequence $\left(p_{d}(n)\right)_{n \in \mathbb{N}}$ for $d=2,3,4,5$.

\section{A class of congruences}

This short section is devoted to the proof of a class of congruences involving partitions into $d$ th powers under certain restrictions. More precisely, let $p_{1}, p_{2} \in \mathbb{N}_{\geq 2}$ and assume that $\left(p_{1}, p_{2}\right)=1$. Let $A_{p_{1}, p_{2}}(n)$ denote the number of partitions into $d$ th powers of integers not divisible by $p_{1}^{d}$ or $p_{2}^{d}$. It is easy to see that the generating function 
for the sequence $\left(A_{p_{1}, p_{2}}(n)\right)_{n \in \mathbb{N}}$ is

$$
\mathcal{A}_{p_{1}, p_{2}}(q)=\sum_{n=0}^{\infty} A_{p_{1}, p_{2}}(n) q^{n}=\prod_{n=1}^{\infty} \frac{\left(1-q^{\left(p_{1} n\right)^{d}}\right)\left(1-q^{\left(p_{2} n\right)^{d}}\right)}{\left(1-q^{n^{d}}\right)\left(1-q^{\left(p_{1} p_{2} n\right)^{d}}\right)} .
$$

We prove the following result. Here, $\mathbb{P}$ denotes the set of primes.

THEOREM 2.1. Let $d \in \mathbb{N}_{+}, n \in \mathbb{N}$ and $p_{1}, p_{2} \in \mathbb{N}_{\geq 2}$. Let $B_{p_{1}, p_{2}}(n)$ denote the number of partitions of $n$ into distinct dth powers not divisible by $p_{2}^{d}$, where each part has one among $p_{1}^{d}-1$ colours, and let $C_{p_{1}, p_{2}}(n)$ denote the number of partitions of $n$ into $d$ th powers not divisible by $p_{2}^{d}$, where each part has one among $p_{1}^{d}-1$ colours.

(1) If $p_{1}=2$ and $p_{2}$ is odd, then $A_{2, p_{2}}(n) \equiv B_{2, p_{2}}(n)(\bmod 2)$.

(2) If $p_{1} \in \mathbb{P}_{\geq 3}$ and $p_{2} \in \mathbb{N}_{\geq 2}, p_{1} \nmid p_{2}$, then, for $n \geq 1$,

$$
\sum_{i=0}^{n} A_{p_{1}, p_{2}}(i) C_{p_{1}, p_{2}}(n-i) \equiv 0\left(\bmod p_{1}\right) .
$$

Proof. From the general theory, it is easy to see that the generating functions of the sequences $\left(B_{p_{1}, p_{2}}(n)\right)_{n \in \mathbb{N}},\left(C_{p_{1}, p_{2}}(n)\right)_{n \in \mathbb{N}}$ are

$$
\begin{aligned}
& \mathcal{B}_{p_{1}, p_{2}}(q)=\sum_{n=0}^{\infty} B_{p_{1}, p_{2}}(n) q^{n}=\prod_{n=1}^{\infty}\left(\frac{1+q^{n^{d}}}{1+q^{\left(p_{2} n\right)^{d}}}\right)^{p_{1}^{d}-1}, \\
& C_{p_{1}, p_{2}}(q)=\sum_{n=0}^{\infty} C_{p_{1}, p_{2}}(n) q^{n}=\prod_{n=1}^{\infty}\left(\frac{1-q^{\left(p_{2} n\right)^{d}}}{1-q^{n^{d}}}\right)^{p_{1}^{d}-1} .
\end{aligned}
$$

We recall a well-known property of formal power series with integer coefficients: if $f \in \mathbb{Z}[[q]]$ and $p$ is a prime number, then $f\left(q^{p^{k}}\right) \equiv f(q)^{p^{k}}(\bmod p)$ for each $k \in \mathbb{N}_{+}$.

Let $p_{1}$ be prime. Using the aforementioned property, we note the chain of modulo $p_{1}$ equivalences

$$
\begin{aligned}
\sum_{n=0}^{\infty} A_{p_{1}, p_{2}}(n) q^{n} & =\prod_{n=1}^{\infty} \frac{\left(1-q^{\left(p_{1} n\right)^{d}}\right)\left(1-q^{\left(p_{2} n\right)^{d}}\right)}{\left(1-q^{n^{d}}\right)\left(1-q^{\left(p_{1} p_{2} n\right)^{d}}\right)} \equiv \prod_{n=1}^{\infty} \frac{\left(1-q^{n^{d}}\right)^{p_{1}^{d}}\left(1-q^{\left(p_{2} n\right)^{d}}\right)}{\left(1-q^{n^{d}}\right)\left(1-q^{\left(p_{2} n\right)^{d}}\right)^{p_{1}^{d}}} \\
& \equiv \prod_{n=1}^{\infty}\left(\frac{1-q^{n^{d}}}{1-q^{\left(p_{2} n\right)^{d}}}\right)^{p_{1}^{d}-1}\left(\bmod p_{1}\right) .
\end{aligned}
$$

If $p_{1}=2$, then

$$
\prod_{n=1}^{\infty}\left(\frac{1-q^{n^{d}}}{1-q^{\left(p_{2} n\right)^{d}}}\right)^{2^{d}-1} \equiv \prod_{n=1}^{\infty}\left(\frac{1+q^{n^{d}}}{1+q^{\left(p_{2} n\right)^{d}}}\right)^{2^{d}-1}=\sum_{n=0}^{\infty} B_{2, p_{2}}(n) q^{n}
$$

and the first result follows by comparison of coefficients in the extreme expressions.

If $p_{1} \geq 3$, then

$$
\prod_{n=1}^{\infty}\left(\frac{1-q^{n^{d}}}{1-q^{\left(p_{2} n\right)^{d}}}\right)^{p_{1}^{d}-1}=\prod_{n=1}^{\infty}\left(\frac{1-q^{\left(p_{2} n\right)^{d}}}{1-q^{n^{d}}}\right)^{1-p_{1}^{d}}=\left(\sum_{n=0}^{\infty} C_{p_{1}, p_{2}}(n)\right)^{-1},
$$


and so

$$
\mathcal{A}_{p_{1}, p_{2}}(q) C_{p_{1}, p_{2}}(q) \equiv 1\left(\bmod p_{1}\right) .
$$

Comparing coefficients on both sides of this congruence gives the second part of the theorem.

REMARK 2.2. If we assume that $p_{1}, p_{2}$ are both primes, then performing the same reasoning as in the proof of the theorem above with respect to the modulus $p_{2}$ instead of $p_{1}$ gives an additional congruence

$$
\mathcal{A}_{p_{1}, p_{2}}(q) C_{p_{2}, p_{1}}(q) \equiv 1\left(\bmod p_{2}\right) .
$$

Thus

$$
\mathcal{A}_{p_{1}, p_{2}}(q) \equiv \frac{a}{C_{p_{1}, p_{2}}(q)}+\frac{b}{C_{p_{2}, p_{1}}(q)}\left(\bmod p_{1} p_{2}\right),
$$

where $a, b$ are the unique solutions of the system of congruences

$$
a \equiv 1\left(\bmod p_{1}\right), \quad a \equiv 0\left(\bmod p_{2}\right), \quad b \equiv 0\left(\bmod p_{1}\right), \quad b \equiv 1\left(\bmod p_{1}\right) .
$$

Consequently,

$$
\sum_{i_{1}+i_{2}+i_{3}=n} A_{p_{1}, p_{2}}\left(i_{1}\right) C_{p_{1}, p_{2}}\left(i_{2}\right) C_{p_{2}, p_{1}}\left(i_{3}\right) \equiv a C_{p_{2}, p_{1}}(n)+b C_{p_{1}, p_{2}}(n)\left(\bmod p_{1} p_{2}\right) .
$$

REMARK 2.3. The number $A_{2, p_{2}}(n)$ has another interpretation: if $D_{p_{2}}(n)$ denotes the number of partitions into $d$ th powers of odd integers in which no part appears more then $p_{2}^{d}-1$ times, then $A_{2, p_{2}}(n)=D_{p_{2}}(n)$. Indeed, this can be deduced from the general theorem concerning partition ideals (see [1, Theorem 8.4$]$ ) or can be directly proved by performing simple manipulations of infinite products. We owe this remark to George Andrews (personal communication, 27 April 2020).

\section{Questions and conjectures concerning the sequence $\left(p_{d}(n)\right)_{n \in \mathbb{N}}$}

Let $d \in \mathbb{N}_{\geq 2}$ be fixed. In this section, we state some questions and conjectures concerning certain aspects of the arithmetic behaviour of functions counting $d$ th power partitions.

Let us write

$$
P_{d}(q)=\prod_{n=1}^{\infty} \frac{1}{1-q^{n^{d}}}=\sum_{n=0}^{\infty} p_{d}(n) q^{n} .
$$

Using the standard method of logarithmic differentiation,

$$
q \frac{P_{d}^{\prime}(q)}{P_{d}(q)}=\sum_{n=1}^{\infty} \frac{n^{d} q^{n^{d}}}{1-q^{n^{d}}}=\sum_{n=1}^{\infty} \sigma^{(d)}(n) q^{n},
$$

where

$$
\sigma^{(d)}(n)=\sum_{k^{d} \mid n} k^{d}
$$


with the usual convention that $\sigma^{(d)}(0)=0$. Simple manipulations give the following recurrence relation satisfied by the sequence $\left(p_{d}(n)\right)_{n \in \mathbb{N}}$ : that is,

$$
n p_{d}(n)=\sum_{i=0}^{n-1} \sigma^{(d)}(i) p_{d}(n-i), \quad p_{d}(0)=1 .
$$

This formula can be used to compute $p_{d}(n)$ in terms of $p_{d}(i)$ for $i<n$. However, even for relatively small values of $n$, the computations are slow. It would be interesting to find a different recurrence formula for $p_{d}(n)$ that allows faster computation for large values of $n$.

For $d=2,3,4,5$, we compute the coefficients $p_{d}(n)$ for $n \leq 10^{5}$, using the following approach. First, we note that

$$
P_{d}(q)-\prod_{i=1}^{\left\lceil 10^{5 / d}\right\rceil} \frac{1}{1-q^{i^{d}}}=O\left(q^{10^{5}+1}\right),
$$

that is, instead of working with the infinite product $P_{d}(q)$, it is enough to work with a rational function. If we write

$$
P_{d, k}(q)=\prod_{i=1}^{k} \frac{1}{1-q^{i^{d}}}=\sum_{n=0}^{\infty} p_{d, k}(n) q^{n},
$$

then $p_{d, k}(n)=p_{d}(n)$ for $n \leq k^{d}$. Note that, for fixed $k, d$, the sequence $\left(p_{d, k}(n)\right)_{n \in \mathbb{N}}$ satisfies a linear recurrence. More precisely, $p_{d, 1}(n)=1$ and, for $k \geq 2$,

$$
p_{d, k}(n)=p_{d, k-1}(n) \text { for } n \leq k^{d}, \quad p_{d, k}(n)=p_{d, k-1}(n)+p_{d, k}\left(n-k^{d}\right) \text { for } n \geq k^{d} .
$$

We used this observation to compute $p_{d}(n)$ for $d=2$ and $n \leq 10^{5}$ and $p_{d}(n)$ for $d=3,4,5$ and $n \leq 10^{6}$. To compute $p_{2}(n)$ for $n \leq 10^{5}$, we take $k=\left\lceil 10^{5 / 2}\right\rceil=317$. Similarly, to compute $p_{d}(n)$ for $d=3,4,5$ for $n \leq 10^{6}$, we take $k=10^{2}, 32,16$, respectively. All computations were performed on an ordinary laptop with 16 GB of memory and an i7 type processor.

Based on our data, we formulate several question and conjectures.

Conjecture 3.1. Let $d \in \mathbb{N}_{\geq 2}$ and $m \in \mathbb{N}_{\geq 2}$ be given and take $r \in\{0, \ldots, m-1\}$. Then there are infinitely many values of $n \in \mathbb{N}$ such that $p_{d}(n) \equiv r(\bmod m)$.

The next question concerns the asymptotic behaviour of the number of solutions of the congruence $p_{d}(n) \equiv r(\bmod m)$, where $m \in \mathbb{N}_{\geq 2}$ and $r \in\{0, \ldots, m-1\}$.

Question 3.2. Let $d \in \mathbb{N}_{\geq 2}$ and $m \in \mathbb{N}_{\geq 2}$ be given and take $r \in\{0, \ldots, m-1\}$. Are the values of $p_{d}(n) \bmod m$ equidistributed modulo $m$ ? More precisely, is it true that

$$
\limsup _{N \rightarrow+\infty} \frac{\#\left\{n \leq N: p_{d}(n) \equiv r(\bmod m)\right\}}{N}=\frac{1}{m} ?
$$

This is a very difficult question. We do not even know any equidistribution modulo $m$ results for the classical partition function $p(n)=p_{1}(n)$ for any $m$. In fact, the expectation is that, for $m$ co-prime to six, the values of $p_{1}(n) \bmod m$ are not 


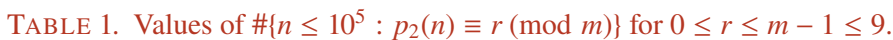

\begin{tabular}{lcccccccccc}
\hline$m \backslash r$ & 0 & 1 & 2 & 3 & 4 & 5 & 6 & 7 & 8 & 9 \\
\hline 2 & 50299 & 49702 & & & & & & & & \\
3 & 33373 & 33249 & 33379 & & & & & & & \\
4 & 25252 & 24695 & 25047 & 25007 & & & & & & \\
5 & 19940 & 20125 & 19971 & 19955 & 20010 & & & & & \\
6 & 16769 & 16454 & 16735 & 16604 & 16795 & 16644 & & & & \\
7 & 14121 & 14272 & 14320 & 14401 & 14257 & 14301 & 14329 & & & \\
8 & 12679 & 12288 & 12496 & 12371 & 12573 & 12407 & 12551 & 12636 & & \\
9 & 11158 & 11081 & 11033 & 10941 & 11186 & 11239 & 11274 & 10982 & 11107 & \\
10 & 10001 & 10025 & 10024 & 9866 & 10085 & 9939 & 10100 & 9947 & 10089 & 9925 \\
\hline
\end{tabular}

TABLE 2. Values of $\#\left\{n \leq 10^{6}: p_{3}(n) \equiv r(\bmod m)\right\}$ for $0 \leq r \leq m-1 \leq 9$.

\begin{tabular}{lcccccccccc}
\hline$m \backslash r$ & 0 & 1 & 2 & 3 & 4 & 5 & 6 & 7 & 8 & 9 \\
\hline 2 & 500013 & 499988 & & & & & & & \\
3 & 333942 & 333563 & 332496 & & & & & & & \\
4 & 250099 & 249905 & 249914 & 250083 & & & & & & \\
5 & 199907 & 200126 & 200490 & 199879 & 199599 & & & & & \\
6 & 167109 & 166685 & 166026 & 166833 & 166878 & 166470 & & & & \\
7 & 142501 & 142721 & 142969 & 143340 & 142937 & 142913 & 142620 & & & \\
8 & 125203 & 124636 & 125023 & 125198 & 124896 & 125269 & 124891 & 124885 & & \\
9 & 111451 & 111275 & 111186 & 111459 & 110992 & 110438 & 111032 & 111296 & 110872 & \\
10 & 100033 & 100134 & 100021 & 99625 & 99713 & 99874 & 99992 & 100469 & 100254 & 99886 \\
\hline
\end{tabular}

equidistributed. However, it is not clear what to expect in our situation because there are no connections to modular forms and Galois representations as in the case of the classical partition function. Our computations of the quantities

$$
\#\left\{n \leq m_{d}: p_{d}(n) \equiv r(\bmod m)\right\}
$$

for $d=2,3,4,5$ seem to confirm Conjecture 3.1 and the equality stated in Question 3.2 (at least, for $m \leq 10$ ); see Tables 1, 2, 3 and 4).

In the context of Euler's classical partition function, $p(n)=p_{1}(n)$, there are plenty of triples $a, b, m$, where $m \in \mathbb{N}_{\geq 5}$ and $a, b \in \mathbb{N}_{+}$, such that $p(a n+b) \equiv 0(\bmod m)$ for all $n \in \mathbb{N}$. This suggests the following question.

Question 3.3. Let $d \in \mathbb{N}_{\geq 2}$ be fixed. Do there exist $m \in \mathbb{N}_{\geq 2}, r \in\{0, \ldots, m-1\}$ and positive integers $a, b$ such that $p_{d}(a n+b) \equiv r(\bmod m)$ for each $n \in \mathbb{N}$ ? 
TABLE 3. Values of $\#\left\{n \leq 10^{6}: p_{4}(n) \equiv r(\bmod m)\right\}$ for $0 \leq r \leq m-1 \leq 9$.

\begin{tabular}{lcccccccccc}
\hline$m \backslash r$ & 0 & 1 & 2 & 3 & 4 & 5 & 6 & 7 & 8 & 9 \\
\hline 2 & 500517 & 499484 & & & & & & & & \\
3 & 333153 & 333474 & 333374 & & & & & & & \\
4 & 250463 & 249010 & 250054 & 250474 & & & & & & \\
5 & 200555 & 199837 & 199524 & 200091 & 199994 & & & & & \\
6 & 166388 & 166699 & 167354 & 166765 & 166775 & 166020 & & & & \\
7 & 143174 & 142713 & 143172 & 142658 & 142908 & 142621 & 142755 & & & \\
8 & 125224 & 124544 & 125595 & 125373 & 125239 & 124465 & 124459 & 125101 & & \\
9 & 111012 & 111100 & 111214 & 111263 & 111238 & 111071 & 110878 & 111136 & 111089 & \\
10 & 100310 & 99810 & 99660 & 99706 & 100135 & 100245 & 100027 & 99864 & 100385 & 99859 \\
\hline
\end{tabular}

TABLE 4. Values of $\#\left\{n \leq 10^{6}: p_{5}(n) \equiv r(\bmod m)\right\}$ for $0 \leq r \leq m-1 \leq 9$.

\begin{tabular}{lcccccccccc}
\hline$m \backslash r$ & 0 & 1 & 2 & 3 & 4 & 5 & 6 & 7 & 8 & 9 \\
\hline 2 & 500386 & 499615 & & & & & & & & \\
3 & 334253 & 332498 & 333250 & & & & & & & \\
4 & 249768 & 249985 & 250618 & 249630 & & & & & & \\
5 & 199971 & 199526 & 200089 & 200380 & 200035 & & & & & \\
6 & 167002 & 166054 & 166940 & 167251 & 166444 & 166310 & & & & \\
7 & 143141 & 142701 & 142907 & 143029 & 142768 & 143046 & 142409 & & & \\
8 & 124187 & 125010 & 125168 & 125302 & 125581 & 124975 & 125450 & 124328 & & \\
9 & 111905 & 111078 & 110740 & 110779 & 111233 & 111095 & 111569 & 110187 & 111415 & \\
10 & 100264 & 99955 & 100250 & 100380 & 100301 & 99707 & 99571 & 99839 & 100000 & 99734 \\
\hline
\end{tabular}

In the range of our calculations, we were unable to find a single quadruple $(m, r, a, b)$ and $d \in\{2,3,4,5\}$ such that $p_{d}(a n+b) \equiv r(\bmod m)$ for $n=0,1 \ldots, 100$. In order to guarantee that $100 a+b \leq 10^{5}$, we considered the range $a \in\{2, \ldots, 999\}$ and $b \in$ $\{0, \ldots, a-1\}$. This may suggest that even if there are quadruplets $(m, r, a, b)$ such that $p_{d}(a n+b) \equiv r(\bmod m)$ for all $n$, they are rare.

A sequence $\left(a_{n}\right)_{n \in \mathbb{N}}$ is convex if $2 a_{n} \leq a_{n-1}+a_{n+1}$ for $n \geq 1$. We formulate the following general conjecture.

Conjecture 3.4. Let $d \in \mathbb{N}_{\geq 2}$. Then there is an integer $N_{d}$ such that, for all $n \geq N_{d}$,

$$
2 p_{d}(n) \leq\left(p_{d}(n-1)+p_{d}(n+1)\right)\left(1-\frac{1}{n^{d}}\right) .
$$

In particular, the sequence $\left(p_{d}(n)\right)_{n \geq N_{d}}$ is convex. 
This conjecture can be seen as a natural generalisation of log-concavity of the classical partition function $p(n)=p_{1}(n)$. We checked that

$$
\begin{gathered}
2 p_{2}(n) \leq\left(p_{2}(n-1)+p_{2}(n+1)\right)\left(1-1 / n^{2}\right) \quad \text { for } n \in\left\{379, \ldots, 10^{5}-1\right\}, \\
2 p_{3}(n) \leq\left(p_{3}(n-1)+p_{3}(n+1)\right)\left(1-1 / n^{3}\right) \quad \text { for } n \in\left\{6769, \ldots, 10^{6}-1\right\}, \\
2 p_{4}(n) \leq\left(p_{4}(n-1)+p_{4}(n+1)\right)\left(1-1 / n^{4}\right) \quad \text { for } n \in\left\{239603, \ldots, 10^{6}-1\right\},
\end{gathered}
$$

that is, we believe that $N_{2}=379, N_{3}=6769, N_{4}=239603$. It seems that the number $N_{5}$ (if it exists) is $\geq 10^{6}$.

A sequence $\left(a_{n}\right)_{n \in \mathbb{N}}$ of positive reals is log-concave if $a_{n}^{2} \geq a_{n-1} a_{n+1}$ for $n \geq 1$, that is, the sequence $\left(-\log a_{n}\right)_{n \in \mathbb{N}}$ is convex. We formulate the following general conjecture.

Conjecture 3.5. Let $d \in \mathbb{N}_{\geq 2}$. Then there is an integer $M_{d}$ such that, for all $n \geq M_{d}$,

$$
p_{d}^{2}(n) \geq p_{d}(n-1) p_{d}(n+1)\left(1+\frac{1}{n^{d}}\right) .
$$

In particular, the sequence $\left(p_{d}(n)\right)_{n \geq M_{d}}$ is log-concave.

We checked that

$$
\begin{array}{cc}
p_{2}^{2}(n) \geq p_{2}(n-1) p_{2}(n+1)\left(1+1 / n^{2}\right) & \text { for } n \in\left\{1086, \ldots, 10^{5}-1\right\}, \\
p_{3}^{2}(n) \geq p_{3}(n-1) p_{3}(n+1)\left(1+1 / n^{3}\right) & \text { for } n \in\left\{15656, \ldots, 10^{6}-1\right\}, \\
p_{4}^{2}(n) \geq p_{4}(n-1) p_{4}(n+1)\left(1+1 / n^{4}\right) & \text { for } n \in\left\{637855, \ldots, 10^{6}-1\right\},
\end{array}
$$

that is, we believe that $M_{2}=1042, M_{3}=15656, M_{4}=637855$. It seems that the number $M_{5}$ (if it exists) is $\geq 10^{6}$.

It is very likely that this conjecture can be resolved using the classical asymptotic formula for $p_{d}(n)$ of Wright (see [10]) or its current improvements. An analogous result for Euler's partition function $p(n)$, that is, the case $d=1$ of the conjecture, was proved by DeSalvo and Pak (see [4]) and recently generalised by Hou and Zhang (see [7]).

\section{References}

[1] G. E. Andrews, The Theory of Partitions (Cambridge University Press, Cambridge, 1998).

[2] G. E. Andrews, 'Partitions: at the interface of $q$-series and modular forms', Ramanujan J. 7 (2003), 385-400.

[3] A. Ciolan, 'Asymptotics and inequalities for partitions into squares', Int. J. Number Theory 16(1) (2020), 121-143.

[4] S. DeSalvo and I. Pak, 'Log-concavity of the partition function', Ramanujan J. 38 (2015), 61-73.

[5] A. Gafni, 'Power partitions', J. Number Theory 163 (2016), 19-42.

[6] G. H. Hardy and S. Ramanujan, 'Asymptotic formulae in combinatory analysis', Proc. Lond. Math. Soc. 2 (1918), 75-115.

[7] Q.-H. Hou and Z.-R. Zhang, ' $r$-log-concavity of partition functions', Ramanujan J. 48 (2019), $117-129$.

[8] G. Tenenbaum, J. Wu and Y.-L. Li, 'Power partitions and saddle-point method', J. Number Theory 204 (2019), 435-445. 
[9] R. C. Vaughan, 'Squares: additive questions and partitions', Int. J. Number Theory 11(5) (2015), $1-43$.

[10] E. M. Wright, 'Asymptotic partition formulae. III. Partitions into $k$-th powers', Acta Math. 63(1) (1934), 143-191.

MACIEJ ULAS, Institute of Mathematics,

Faculty of Mathematics and Computer Science,

Jagiellonian University, Łojasiewicza 6, 30 - 348 Kraków, Poland

e-mail: maciej.ulas@uj.edu.pl 